\title{
CLOSED GEODESICS ON A RIEMANN SURFACE WITH APPLICATION TO THE MARKOV SPECTRUM
}

\author{
A. F. BEARDON, J. LEHNER AND M. SHEINGORN ${ }^{1}$
}

\begin{abstract}
This paper determines those Riemann surfaces on which each nonsimple closed geodesic has a parabolic intersection - that is, an intersection in the form of a loop enclosing a puncture or a deleted disk. An application is made characterizing the simple closed geodesic on $H / \Gamma(3)$ in terms of the Markov spectrum.

The thrust of the situation is this: If we call loops about punctures or deleted disks boundary curves, then if the surface has "little" topology, each nonsimple closed geodesic must contain a boundary curve. But if there is "enough" topology, there are nonsimple closed geodesics not containing boundary curves.
\end{abstract}

1. Let $R$ be a Riemann surface of genus $g$ with $k$ punctures and $d$ disks removed; $k, d \geq 0, k+d>0$. In this paper we consider the closed geodesics on $R$.

The method used is to represent $R$ as a quotient, $R=H / \Gamma$, where $H=\{z=$ $x+i y: y>0\}$ and $\Gamma$ is a fuchsian group acting on $H$. Let $\pi: H \rightarrow H / \Gamma$ be the projection map. We assume $(H, \pi)$ is an unramified covering, so $\Gamma$ has no elliptic elements. For most of this paper we shall assume $k>0$ and $d=0$; the remaining cases are considered in $\S 5$. Each hyperbolic axis projects to a closed geodesic in $R$. Each closed geodesic $\alpha$ in $R$ lifts to a conjugacy class $[\alpha]$ in $\Gamma$ and under known conditions $[\alpha]$ is hyperbolic.

Let $\gamma \in \Gamma$ be hyperbolic with axis $A_{\gamma}$. From now on we assume $\gamma$ primitive; i.e., $\gamma$ generates the stabilizer of $A_{\gamma}$. It is easily seen that

$$
\pi\left(A_{\gamma}\right) \text { is simple iff } A_{\gamma} \cap \beta A_{\gamma}=\varnothing \text { for all } \beta \in \Gamma-\langle\gamma\rangle \text {. }
$$

We write $A_{\gamma} \wedge \beta A_{\gamma}$ to mean that $A_{\gamma} \cap \beta A_{\gamma}=\{z\}$ for some $z$ in $H$. Thus

$$
\begin{aligned}
& \pi\left(A_{\gamma}\right) \text { is nonsimple (= self-intersecting) iff } \\
& A_{\gamma} \wedge \beta A_{\gamma} \text { for some } \beta \in \Gamma-\langle\gamma\rangle .
\end{aligned}
$$

Since $\alpha A_{\gamma}=A_{\alpha \gamma \alpha^{-1}}$, conjugate elements are both simple or both nonsimple. We use the phrase a hyperbolic $\gamma$ is simple (nonsimple) to mean $\pi\left(A_{\gamma}\right)$ is simple (nonsimple).

It is readily checked that if $\gamma$ is nonsimple, we can choose $\beta$ in (1.1) to be hyperbolic; indeed, we may replace $\beta$ by $\beta \gamma^{n}$ for large $n$. However, it is not always possible to choose $\beta$ to be parabolic, and in this connection we prove the following result.

Received by the editors Jaunary $8,1985$.

1980 Mathematics Subject Classification (1985 Revision). Primary 30F35; Secondary 11 J06.

${ }^{1}$ Third author partially supported by NSF and PSC-CUNY grants. 
THEOREM 1.1. Let $R$ and $\Gamma$ be as above and let $d=0$. Then it is possible to select $\beta$ in (1.1) to be parabolic for every nonsimple $\gamma$ if and only if $g=0$ and $k=3$ or 4 .

The area formula for a fushsian group shows that $g=0$ only if $k \geq 3$. For example, $\Gamma(2)$ and $\Gamma(3)$ have $g=0$ and $k=3$ and 4 , respectively, so Theorem 1.1 applies to these groups. (Here $\Gamma(n)$ is the subgroup of the modular group consisting of all modular matrices $V \equiv \pm I(\bmod n)$.)

In $\S 2$ we develop a connection with the Markov spectrum of diophantine approximation. To introduce the Markov spectrum, let $\theta$ be a real irrational and define

$$
\begin{aligned}
& M(\theta)=\sup _{c>0}\left\{c:\left|\theta-\frac{p}{q}\right|<\frac{1}{c q^{2}}\right. \text { for infinitely many reduced } \\
& \left.\qquad \text { fractions } \frac{p}{q}, q>0\right\} .
\end{aligned}
$$

We are interested only in the range $M(\theta)<3$, and in this range $M(\theta)$ assumes only a countably infinite set of values $M_{\nu}$. The set $\left\{M_{\nu}\right\}$, ordered by magnitude $\left(M_{1} \leq M_{2} \leq \cdots\right)$, is called the Markov spectrum and abbreviated to MS. Moreover, $M_{\nu} \rightarrow 3$ and, for each $\nu$, there is a real quadratic irrational $\xi_{\nu}$ such that $M_{\nu}=$ $M\left(\xi_{\nu}\right)$. The $\xi_{\nu}$ are known explicitly, and there is a primitive hyperbolic matrix $\gamma_{\nu}$ in $\Gamma(3)$ that fixes $\xi_{\nu}$ and its conjugate $\xi_{\nu}^{\prime}$. (See $\S 2$ for details.)

THEOREM 2.1. Let $\gamma \in \Gamma(3)$ be hyperbolic with fixed points $\xi_{\gamma}, \xi_{\gamma}^{\prime}$. Then $\pi\left(A_{\gamma}\right)$ is simple if and only if the fixed points $\xi_{\gamma}, \xi_{\gamma}^{\prime}$ of $\gamma$ satisfy $M\left(\xi_{\gamma}\right)=M\left(\xi_{\gamma}^{\prime}\right)<3$.

This result, in particular, specifies the lengths of the simple closed geodesics in $H / \Gamma(3)$. Indeed, if $\gamma$ is a transformation for which $M\left(\xi_{\gamma}\right)<3$, then $\pi\left(A_{\gamma}\right)$ has length $L$ where

$$
\operatorname{trace} \gamma=2 \cosh \left(\frac{1}{2} L\right)
$$

Using an estimate of C. Gurwood given in [5], this leads to

COROLlaRY 2.2. Let $N_{s}(X)=\#$ simple closed geodesics on $H / \Gamma(3)$ with length $\leq X\}$. Then $X \ll N_{s}(X) \ll X^{2}$.

This work grew out of the characterization of the simple closed geodesics on $H / \Gamma(3)$ given by Lehner and Sheingorn in [4]. $H / \Gamma(3)$ is a four-times punctured sphere and the original proof of the result in [4] relied on the geometry of that surface. Beardon pointed out that these geometric arguments could largely be replaced by topological ones and that these new arguments applied to more general Riemann surfaces. He also saw that the new arguments had converses-that is, they pertained exactly to the surfaces with $g=0$ and $k+d=3$ or 4 .

At about the same time, Haas was achieving his characterization of the simple geodesics on punctured tori [3]. He does this using binary quadratic forms studied by Cohn [1] and Schmidt [6]. For $H / \Gamma^{\prime}$, his characterization (in the closed case) is the same as that of [4]. His paper is more general than [4] in two ways: (i) He treats the closure of the set of simple geodesics (closed or infinite); (ii) his results apply to the signature class $(1, \infty)$, not just $H / \Gamma^{\prime}$. The article of Series [7] is a nice 
exposition of the work of Cohn, Schmidt and (decisively) Haas on simple closed geodesics on $H / \Gamma^{\prime}$.

After Haas personally communicated his work to Sheingorn, the latter realized that the characterizations were the same for $H / \Gamma(3)$ and $H / \Gamma^{\prime}$ because they both stemmed from a characterization of the simple closed geodesics on $H / \Gamma^{3}$ - the crucial point being that $\Gamma^{3}$ contains both $\Gamma^{\prime}$ and $\Gamma(3)$ as "large" subgroups. Again this can be generalized, à la Haas, to entire signature classes [8].

2. In this section we prove Theorem 2.1 and Corollary 2.2 , which connect closed simple geodesics in $H / \Gamma(3)$ with the Markov spectrum (MS). We include a brief synopsis of known facts about MS; for a fuller account see [2, pp. 29-33].

In (1.2) and the following lines we defined MS to be the set of values $\left\{M_{\nu}\right\}$ assumed by the Markov function $M(\theta)$ in the range $M(\theta)<3$. In order to calculate $M_{\nu}$ we introduce Markov triples. A triple of positive integers $(x, y, z)$ is called a Markov triple if

$$
x^{2}+y^{2}+z^{2}=3 x y z, \quad 1 \leq x \leq y \leq z
$$

The first triples are $(1,1,1),(1,1,2),(1,2,5), \ldots$, and the rest can be recursively generated. Order the triples by the size of $z$, so that $1=z_{1} \leq 2=z_{2} \leq \cdots \leq$ $z_{\nu} \leq \cdots$. With each triple $\left(x_{\nu}, y_{\nu}, z_{\nu}\right)$ there is associated a pair of real quadratic conjugates

$$
\theta_{\nu}, \theta_{\nu}^{\prime}=\frac{1}{2}+\frac{y_{\nu}}{x_{\nu} z_{\nu}} \pm \frac{1}{2}\left(9-\frac{4}{z_{\nu}^{2}}\right)^{1 / 2}, \quad \nu \geq 1
$$

It is a known theorem that $M\left(\theta_{\nu}\right)=M\left(\theta_{\nu}^{\prime}\right)=M_{\nu}$, and that

$$
M\left(\theta_{\nu}\right)=M_{\nu}=\left|\theta_{\nu}-\theta_{\nu}^{\prime}\right|=\left(9-4 / z_{\nu}^{2}\right)^{1 / 2}
$$

We have $M_{1}=5^{1 / 2}, M_{2}=8^{1 / 2}, M_{3}=(221)^{1 / 2} / 5, \ldots, \rightarrow 3$.

Next, introduce the equivalence relation:

$$
\begin{aligned}
& \theta \sim \psi \text { if } \psi=(a \theta+b) /(c \theta+d) \text { with integers } \\
& a, b, c, d \text { and } a d-b c= \pm 1 .
\end{aligned}
$$

Then

$$
\theta \sim \psi \Rightarrow M(\theta)=M(\psi) \text {; in particular, } M(V \theta)=M(\theta) \text { for } V \in \Gamma(1) .
$$

To understand why this is true, we adopt the usual notation for the regular continued fraction (CF)

$$
\theta=a_{0}+\frac{1}{a_{1}+\frac{1}{a_{2}+\cdots}}=\left[a_{0}, a_{1}, a_{2}, \ldots\right] .
$$

(Here $a_{i} \geq 1$ for $i \geq 1$.) Then it is well known that $\theta \sim \psi$ means that the CF expansions of $\theta$ and $\psi$ agree from some point on. That is, there exist $n_{0}$ and $m_{0}$ such that $a_{n_{0}+k}=b_{m_{0}+k}, k=0,1,2, \ldots$ (Here $\psi=\left[b_{0}, b_{1}, b_{2}, \ldots\right]$.) Next, it can be shown that [2, p. 29]

$$
M(\theta)=\varlimsup_{k}\left\{\left[a_{k+1}, a_{k+2}, \ldots\right]+\left[0, a_{k}, a_{k-1}, \ldots, a_{1}\right]\right\} .
$$

Given this, (2.2) is clear. 
We can associate the MS to hyperbolic elements of $\Gamma(3)$. For each $\nu$ there is a $\gamma_{\nu} \in \Gamma(3)$ whose fixed points are $\xi_{\gamma_{\nu}}=\theta_{\nu}, \xi_{\gamma_{\nu}}^{\prime}=\theta_{\nu}^{\prime}$. Namely, dropping the subscript $\nu$, let $\zeta=1$ if $z$ is odd; otherwise $\zeta=2$. Define

$$
B=\left(\begin{array}{cc}
\left(N+x(2 y+x z) M \zeta^{-1}\right) / 2 & \left(2 x^{2} z-4 x y+z\right) M_{\zeta^{-1}} \\
x^{2} z M \zeta^{-1} & \left(N-x(2 y+x z) M \zeta^{-1}\right) / 2
\end{array}\right)
$$

where $M>0$ is the smallest integral solution of the Pell equation

$$
x^{4}\left(9 z^{2}-4\right) \zeta^{-2} M^{2}+4=N^{2} .
$$

Then it can be shown that $B$ is the $\Gamma(1)$-primitive matrix fixing $\xi, \xi^{\prime}$. Moreover, $B \in \Gamma(3)$ if $3 \mid M$; otherwise $B^{2} \in \Gamma(3)$. But the first case never occurs, so $B^{2}$ is the $\Gamma(3)$-primitive matrix fixing $\xi, \xi^{\prime}$. (See $[4,8]$.)

Let $\mathcal{M}=\left\{\gamma \in \Gamma(3): M\left(\xi_{\gamma}\right)<3\right\}$, so Theorem 2.1 may be restated as follows: Let $\gamma \in \Gamma(3)$ be hyperbolic. Then $\pi\left(A_{\gamma}\right)$ is simple if and only if $\gamma \in \mathcal{M}$. Note that $\gamma \in \mathcal{M} \Rightarrow V \gamma V^{-1} \in \mathcal{M}, V \in \Gamma(1)$. Indeed $V \gamma V^{-1} \in \Gamma(3)$ by normality of $\Gamma(3)$ in $\Gamma(1)$. And since $\xi_{V \gamma V^{-1}}=V \xi_{\gamma}, M\left(\xi_{V \gamma V^{-1}}\right)=M\left(V \xi_{\gamma}\right)=M\left(\xi_{\gamma}\right)<3$. That is, the conjugacy class of $\gamma$ in $\Gamma(1)$ belongs to $\mathcal{M}$ if $\gamma \in \mathcal{M}$.

We shall now prove Theorem 2.1. Suppose $\pi\left(A_{\gamma}\right)$ is nonsimple; we wish to show that $\gamma \notin \mathcal{M}$. By Theorem 1.1 and the normality of $\Gamma(1)$, there is a $\delta \in \Gamma(3)$ conjugate to $\gamma$ in $\Gamma(1)$ for which $A_{\delta} \wedge S^{3} A_{\delta}$; i.e., $\left|\xi_{\delta}-\xi_{\delta}^{\prime}\right|>3$. Here $S=\left(\begin{array}{ll}1 & 1 \\ 0 & 1\end{array}\right)$. Denote the periodic continued fraction $\left[a_{0}, \ldots, a_{\mu-1}, b_{\mu}, b_{\mu+1}, \ldots, b_{\mu+k-1}, b_{\mu}, \ldots, b_{\mu+k-1}, \ldots\right]$ by $\left[a_{0}, \ldots, a_{\mu-1}, \overline{b_{\mu}, \ldots, b_{\mu+k-1}}\right]$. By translation in $\Gamma(1)$ we may assume $-1<$ $\xi_{\delta}^{\prime}<0$. Then $\xi_{\delta}>\xi_{\delta}^{\prime}+3>1$. It is well known that under these conditions $\mathrm{CF} \xi_{\delta}$ is pure periodic $[5$, p. 75$]$. Let $\xi_{\delta}=\left[\overline{b_{0}, b_{1}, \ldots, b_{k-1}}\right]$ for a $k \geq 1$; then $-1 / \xi_{\delta}^{\prime}=\left[\overline{b_{k-1}, \ldots, b_{1}}\right]$ by $[5$, p. 76]. Set

$$
m_{\mu}=\left[\overline{b_{\mu}, b_{\mu+1}, \ldots, b_{\mu+k-1}}\right]+\left[0, \overline{b_{\mu-1}, b_{\mu-2}, \ldots, b_{\mu-k}}\right], \quad \mu \geq k .
$$

By periodicity $m_{\mu}=m_{\mu+k}$, so we may restrict $\mu$ to the range $k+1 \leq \mu \leq 2 k$.

Moreover,

$$
M\left(\xi_{\delta}\right)=\varlimsup_{\mu \rightarrow \infty}\left\{\left[b_{\mu}, b_{\mu+1}, \ldots\right]+\left[0, b_{\mu-1}, \ldots, b_{1}\right]\right\} .
$$

Now for $\mu=n k+\nu, 0 \leq \nu<k$,

$$
\left[0, b_{\mu-1}, \ldots, b_{1}\right]=\left[0, B, \ldots, B, b_{\nu-1}, \ldots, b_{1}\right],
$$

where $B$ is the block $b_{\mu-1}, \ldots, b_{\mu-k}$ and there are $n B$ 's. Hence as $\mu \rightarrow \infty$, i.e., $n \rightarrow \infty$, the right member tends to $\left[0, \overline{b_{\mu-1}, \ldots, b_{\mu-k}}\right]$, so

$$
M\left(\xi_{\delta}\right)=\varlimsup_{\mu \rightarrow \infty} m_{\mu}=\max _{k+1 \leq \mu \leq 2 k} m_{\mu}
$$

Hence for $n$ sufficiently large,

$$
3<\xi_{\delta}-\xi_{\delta}^{\prime}=m_{k} \leq \max _{\mu} m_{\mu}=M\left(\xi_{\delta}\right),
$$

implying $M\left(\xi_{\gamma}\right)=M\left(\xi_{\delta}\right)>3$. That is, $\gamma \notin \mathcal{M}$, as asserted.

Conversely, assume $\gamma \notin \mathcal{M}$. Then $M\left(\xi_{\gamma}\right) \geq 3$; in fact $M\left(\xi_{\gamma}\right)>3$, since $\xi_{\gamma}$ is a quadratic irrational and $M(\theta)$ cannot equal 3 for any quadratic irrational $\theta[2, \mathrm{p}$. 32]. Hence

$$
\left|\xi_{\gamma}-p_{n} / q_{n}\right|<1 /(3+h) q_{n}^{2}, \quad\left(p_{n}, q_{n}\right)=1,
$$

for some $h>0$, on a sequence $q_{n} \rightarrow \infty$. 
Write $V_{n}=\left(q_{n}^{\prime},-p_{n}^{\prime}: q_{n},-p_{n}\right) \in \Gamma(1)$. Then with $\xi_{\gamma}=\xi, \xi_{\gamma}^{\prime}=\xi^{\prime}$

$$
\begin{aligned}
\left|V_{n} \xi-V_{n} \xi^{\prime}\right| & =\frac{\left|\xi-\xi^{\prime}\right|}{q_{n}^{2}\left|\xi-p_{n} / q_{n}\right|\left|\xi^{\prime}-p_{n} / q_{n}\right|} \\
& >\frac{(3+h)\left|\xi-\xi^{\prime}\right|}{\left|\xi^{\prime}-p_{n} / q_{n}\right|} \geq \frac{(3+h)\left|\xi-\xi^{\prime}\right|}{\left|\xi^{\prime}-\xi\right|+\left|\xi-p_{n} / q_{n}\right|} \\
& >\frac{3+h}{1+1 / 3 q_{n}^{2}\left|\xi-\xi^{\prime}\right|}>3
\end{aligned}
$$

for $n \geq n_{0}$. For $V=V_{n_{0}}$ we have

$$
\left|V \xi_{\gamma}-V \xi_{\gamma}^{\prime}\right|>3
$$

Next, set $\beta=V^{-1} S^{3} V$, a parabolic element in $\Gamma(3)$. Since $V \xi_{\gamma}, V \xi_{\gamma}^{\prime}$ are the fixed points of $V A_{\gamma} V^{-1},(2.5)$ gives $A_{V \gamma V^{-1}} \wedge S^{3} A_{V \gamma V^{-1}}$. But $A_{V \gamma V^{-1}}=V A_{\gamma}$, so $A_{\gamma} \wedge V^{-1} S^{3} V A_{\gamma}$, i.e., $A_{\gamma} \wedge \beta A_{\gamma}$. By (1.1), $\pi\left(A_{\gamma}\right)$ is not simple. This completes the proof.

We turn now to the proof of Corollary 2.2. Assume that $\gamma$ is a primitive hyperbolic in $\mathrm{SL}(2, \mathrm{Z})$ with positive trace and of the form (2.3) corresponding to the Markov triple $(x, y, z)$. Using (1.3), we have

$$
3 z / 4 \leq\left[x^{2}\left(9 z^{2}-4\right) \varsigma^{-2} M^{2}+4\right]^{1 / 2} / \zeta=\operatorname{trace}(\gamma) \leq e^{L / 2}+1,
$$

so the number of $\gamma$ with $L \leq X$ is not more than the number of $\gamma$ with $z \leq 2 e^{X / 2}$. Using the estimate of this by C. Gurwood, noted in [5], we obtain $N_{s}(X) \ll X^{2}$ with an explicit constant available.

To obtain the lower bound, we simply estimate the number of $\gamma$ with $L \leq X$, which, in addition, correspond to a triple $(1, y, z)$. We can generate a sequence $\gamma_{n}$ of elements with distinct traces of the triples $\left(1, y_{n+1}, z_{n+1}\right)=\left(1, z_{n}, 3 z_{n}-y_{n}\right)$, so $z \leq 3^{n}$. Moreover, we have, by (2.4),

$$
\left(9 z_{n}^{2}-4\right)(M / \varsigma)^{2}+4=N^{2}
$$

which has the fundamental solution $M=\varsigma, N=3 z_{n}$. Thus $\operatorname{trace}\left(\gamma_{n}\right)=3 z_{n} / \varsigma$. If $A=(\log \varsigma) /(\log 9)-1, L_{n}=L\left(\gamma_{n}\right)$, then (1.3) yields

$$
\frac{1}{2} e^{L_{n} / 2} \leq 3^{n+1} / \varsigma \text {. }
$$

If we choose any $n<(X+\log \varsigma) /(\log 9)-1$, then we have that $L_{n}<X$. These values of $n$, then, give distinct $\gamma_{n}$ with $L_{n}<X$, and so

$$
N_{s}(X)>X(\log 9)-1 \text {. }
$$

This completes the proof of Corollary 2.2.

3. In this section we establish the positive assertion only, namely that if $R$ $(=H / \Gamma)$ has genus zero and three or four punctures, then for every nonsimple hyperbolic $\gamma$ in $\Gamma$ there is a parabolic $\beta$ in $\Gamma$ with $\beta\left(A_{\gamma}\right)$ crossing $A_{\gamma}$. We begin by selecting a nonsimple hyperbolic $\gamma$ in $\Gamma$, and there is no loss of generality in supposing that $\gamma$ is primitive. Next we make some preliminary remarks about the axis $A_{\gamma}$ and its projection $\pi\left(A_{\gamma}\right)$ on $R$. As $A_{\gamma}$ is topologically the real line, we can define in a natural way the order relations $<$ and $\leq$ on $A_{\gamma}$, so, for example, if $z$ is 
on $A_{\gamma}$, then $z<\gamma(z)$. The meaning of $[z, w],(z, w),[z, w)$ and $(z, w]$ as intervals on $A_{\gamma}$ is self-evident.

Suppose now that the axis $A_{\gamma}$ contains a nontrivial segment $[z, \alpha z]$ with $\alpha$ in $\Gamma$; thus $c=\pi([z, \alpha z])$ is a closed curve on $R$. If $c$ is homotopic in $R$ to a point of $R$, then, by the Monodromy Theorem, the lift from $z$, namely $[z, \alpha z]$, is closed. Since $\Gamma$ has no elliptics, $\alpha$ must then be the identity, which is false. We record this result as

$$
\text { no closed subarc of } \pi\left(A_{\gamma}\right) \text { is homotopic in } R \text { to a point of } R \text {. }
$$

Next suppose that the axis $A_{\gamma}$ contains a nontrivial segment $[z, \alpha z]$ with the closed curve $c=\pi([z, \alpha z])$ freely homotopic in $R$ to some curve $c^{\prime}$ in a disc $N$ (on the sphere $S=R \cup$ (punctures)) containing exactly one puncture. The lift of $N$ is a maximal set of distinct $\Gamma$-equivalent open horocycles. If the hypothesis is true for some such $N$, then it is true for all smaller discs, and by taking $N$ sufficiently small, these distinct horocycles are pairwise disjoint. The (connected) lift $\sigma^{\prime}$ of $c^{\prime}$ (from any appropriate point) therefore lies in precisely one, say $Q$, of these horocycles. This choice of $N$, however, means that if $\beta$ is in $\Gamma$ then $\beta(Q)=Q$ precisely when $\beta$ is parabolic (or the identity) and stablizes $Q$. We deduce that the endpoints of $\sigma^{\prime}$ are, say, $w$ and $\beta w$ with $\beta$ parabolic or the identity. It is well kown that as $c$ and $c^{\prime}$ are freely homotopic, the endpoints of any lift of $c$ must be of the form $w^{\prime}$ and $\beta^{\prime} w^{\prime}$, say, where $\beta^{\prime}$ is conjugate to $\beta$. Now (3.1) prevents $\beta$ from being the identity, for if it is then so is $\beta^{\prime} ; \sigma^{\prime}$ is then homotopic to a point in $Q$, and consequently $c^{\prime}$, and hence $c$, is homotopic to a point of $R$. We deduce the following:

if a closed subarc of $\pi\left(A_{\gamma}\right)$ is freely homotopic to a curve in a disc of $S$ which contains exactly one puncture, then $\beta\left(A_{\gamma}\right) \cap A_{\gamma} \neq \varnothing$ for some parabolic $\beta$ in $\Gamma$.

Observe that the conclusion of (3.2) is the result we are seeking.

Next, suppose that, for some $\alpha$ and $\beta$ in $\Gamma, A_{\gamma}$ contains two nontrivial segments $[z, w]$ and $[\alpha z, \beta w]$ with $\pi$-images $c$ and $c^{\prime}$ respectively such that $c$ and $c^{\prime}$ are homotopic in $R$. Note that $c^{\prime}=\pi\left(\left[z, \alpha^{-1} \beta w\right]\right)$. By the Monodromy Theorem, the lifts of $c$ and $c^{\prime}$ from $z$ have the same endpoint, so $\alpha=\beta$. Then $\alpha$ maps $A_{\gamma}$ (the geodesic through $z$ and $w$ ) to itself (the geodesic through $\alpha z$ and $\alpha w$ ); hence, as $\gamma$ is primitive, $\alpha \in\langle\gamma\rangle$. This shows that

$$
\begin{aligned}
& \text { no two distinct nontrivial segments }[z, w] \text { and } \\
& {[\alpha z, \beta w] \text { of a fundamental segment of } A_{\gamma} \text { have }} \\
& \text { homotopic } \pi \text {-images in } R \text {. }
\end{aligned}
$$

Our final remark guarantees the existence of simple loops in $A_{\gamma}$. Consider a fundamental segment $[z, \gamma z]$ of $A_{\gamma}$. By assumption, its projection is a nonsimple loop, so there exist equivalent points $z_{1}$ and $z_{1}^{\prime}$ with $z \leq z_{1}<z_{1}^{\prime}<\gamma z$. Write $z_{1}^{\prime}=\gamma_{1} z_{1}$ and repeat the argument for $\left[z_{1}, \gamma_{1} z_{1}\right]$. This process must terminate; else we could find a sequence of pairwise disjoint equivalent points in $[z, \gamma z]$. Using discreteness, one can show that infinitely many of these must be paired by the same element, say $\delta$, of $\Gamma$. Thus (as in the proof of $(3.3)$ ) $\delta$ is in $\langle\gamma\rangle$, and this is clearly false. Thus we have proved

$$
\pi\left(A_{\gamma}\right) \text { contains a simple closed subarc. }
$$


CASE I

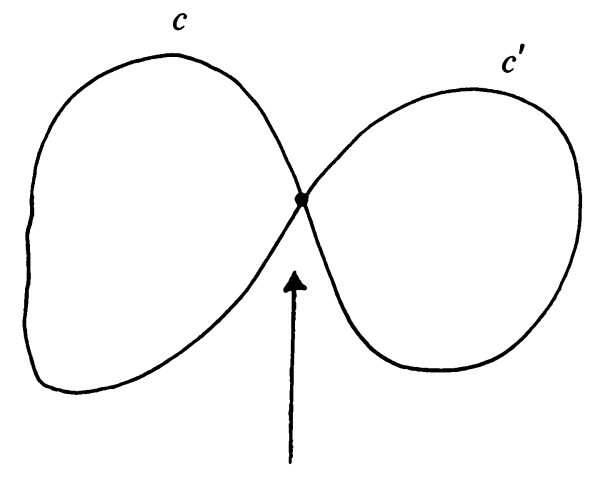

$$
\pi(z)=\pi(\alpha(z))=\pi(w)=\pi(\beta(w))
$$

\section{FIGURE 1}

We can now complete the proof of Theorem 1.1. First, $\pi\left(A_{\gamma}\right)$ contains a simple closed subarc $c$. Now $c$ is a Jordan curve on the sphere $S$ and so has two complementary Jordan domains $D$ and $D^{\prime}$. By (3.1), $D$ and $D^{\prime}$ each contain at least one puncture. If there are exactly three punctures (i.e., if $k=3$ ), then one of $D$ and $D^{\prime}$ contains exactly one puncture, and the conclusion follows from (3.2).

If $k=4$ (which we now assume), then $D$ and $D^{\prime}$ may each contain two punctures, and we need to refine this argument. As $\pi\left(A_{\gamma}\right)$ contains a simple closed subarc, we can construct a fundamental segment $[z, \gamma z]$ of $A_{\gamma}$ containing a point $\alpha z$ (with $\alpha$ in $\Gamma)$ with $\pi 1-1$ on $(z, \alpha z]$. The simple closed subarc is $\pi([z, \alpha z])$. Observe now that $(z, \alpha z] \subset(z, \gamma z)$ and that $\pi$ is $1-1$ on $(z, \alpha z]$ but not on $(z, \gamma z]$ (else $\gamma$ is simple). It follows that there is a first point $w^{\prime}$ after $\alpha z$ and before $\gamma z$ which is equivalent to some point $w$ in $\left(z, w^{\prime}\right)$. Write $w^{\prime}=\beta w$. Thus we have

$$
z<\alpha z<\beta w<\gamma z ; \quad z<w<\beta w
$$

Three possibilities now arise, and we shall give a proof in each case. The easiest is

Case I: $w=\alpha z$ (Figure 1). Our assumptions imply that

$$
c=\pi([z, \alpha z])=\pi([z, w]) \quad \text { and } \quad c^{\prime}=\pi([\alpha z, \beta w])=([w, \beta w])
$$

are simple closed subarcs of $\pi\left(A_{\boldsymbol{\gamma}}\right)$ which meet at one point only, namely the common projection of $z, \alpha z, w$ and $\beta w$. Thus $c \cup c^{\prime}$ is a "figure eight" curve on $S$, and this has three complementary domains on $S$. Two of these are discs (bounded by $c$ and by $c^{\prime}$ respectively); the third is a topological disc bounded by $c \cup c^{\prime}$. If our assertion is false, then these must contain at least 2,2 and 1 punctures respectively (see (3.1), (3.2) and (3.3)), and so $k \geq 5$. As $k=4$, the proof for Case I is complete.

It is worth noting that the figure eight curve (or a variant of it) dominates all stages of the proof of Theorem 1, and the reader may find it helpful to bear this in mind. 
CASE II

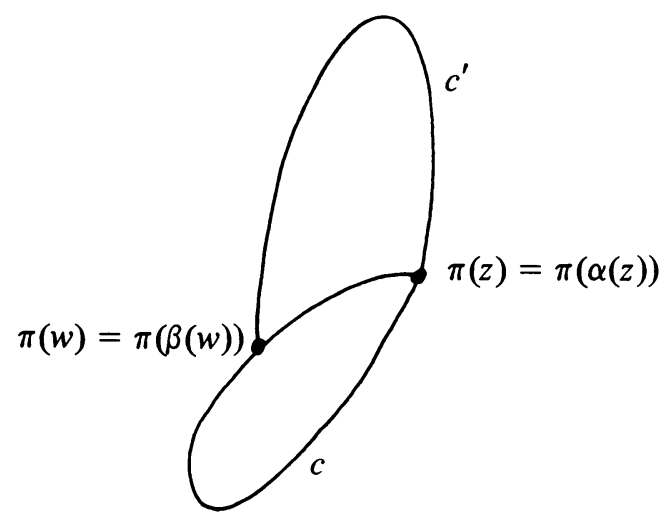

FIGURE 2

CASE III

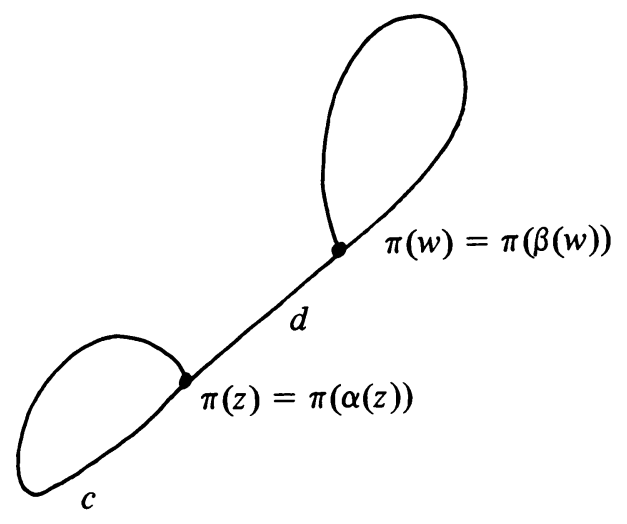

FIGURE 3

Case $\Pi: w \in(z, \alpha z)$ (Figure 2).

The curve $c=\pi([z, \alpha z])$ is a Jordan curve which contains the two distinct points $\pi(z)$ and $\pi(w)$. The curve $c^{\prime}=([\alpha z, \beta w])$ joins these two points. Moreover, by assumption, $c^{\prime}$ is simple and does not meet $c$ at any points other than $\pi(z)$ and $\pi(w)$. Thus the complement of $c$ in $S$ is the union of two discs, say $V$ and $W$, and $c^{\prime}$ is a simple cross-cut of $W$, say. It follows that the complement of $c \cup c^{\prime}$ on $S$ is the disjoint union of three Jordan domains bounded respectively by the $\pi$-images of $[z, \alpha z],[z, w] \cup[\alpha z, \beta w],[w, \beta w]$. By (3.1) to (3.3), the result can only fail if these domains contain 2, 1, 2 punctures respectively, and this cannot be so.

Case III: $w \in(\alpha z, \beta w)$ (Figure 3).

The $\pi$-image of $[z, \beta w]$ consists of the disjoint union of the simple loop $c=$ $\pi([z, \alpha z])$, the simple arc $d=\pi((\alpha z, w))$ and the simple loop $c^{\prime}=\pi([w, \beta w])$. By construction, $d$ joins the first (and last) point of $c$ to that of $c^{\prime}$. Thus the complement on $S$ of these three curves consists of three Jordan domains, say $U$ (bounded by $c$ ), $V$ (bounded by $c^{\prime}$ ) and $W$ (a simply connected domain bounded by the two curves $c \cup d$ and $d \cup c^{\prime}$, each joining $\pi(z)$ to $\left.\pi(w)\right)$. Again, if the result fails, then these must contain at least 2, 2 and 1 punctures, and the proof of the "if" part of Theorem 1.1 is complete. 
4. We now complete the proof of Theorem 1.1. Recall that $R(=H / \Gamma)$ is a Riemann surface of genus $g$ with $k$ punctures. Since $\Gamma$ has no elliptic elements, $\Gamma$ is isomorphic to the fundamental group $\Gamma^{*}$ of $R$, and in view of this we shall regard $\Gamma$ and $\Gamma^{*}$ as being the same group; for example, we shall speak of parabolic elements of $\Gamma^{*}$. Likewise, any homomorphism $\theta: \Gamma^{*} \rightarrow \mathbf{Z}$ may be regarded as being defined on $\Gamma$ : if $x$ in $\Gamma^{*}$ corresponds to $\gamma$ in $\Gamma$, then $\theta(x)=\theta(\gamma)$.

In this section we verify that if $g \geq 1$, or if both $g=0$ and $k \geq 5$, then $\Gamma$ contains a primitive nonsimple hyperbolic $\gamma$ such that $\beta\left(A_{\gamma}\right) \cap A_{\gamma}=\varnothing$ for every parabolic $\beta$ in $\Gamma$. The proofs for $g=0$ and for $g \geq 1$ follow a similar argument, which we now describe, but are different in their details. We construct a closed curve $x$ on $R$ (actually a figure eight) with certain specified geometric properties. Now $x$ represents an element of $\Gamma^{*}$, and this lifts from $z$ to $\gamma z$ for some $\gamma$ in $\Gamma$. Because of the geometric properties of $x, \gamma$ will possess the stated properties.

The proof for $g=0, k \geq 5$. We assume that $k=5$ (only a trivial change is needed if $k>5$ ), and without loss of generality we may assume that $R=$ $S-\left\{\infty, w_{1}, w_{2}, w_{3}, w_{4}\right\}$, where $S$ is the sphere and the punctures are at $\infty$ and $w_{j}$. Choosing a base point $w$ in $R$ for the fundamental group of $R$, construct two simple closed curves $a$ and $b$ (each starting and ending at $w$ ) with the following properties (in which $N(x, v)$ denotes the winding number of $x$ about $v$ ):

(i) $N\left(a, w_{1}\right)=N\left(a, w_{2}\right)=1, N\left(a, w_{3}\right)=N\left(a, w_{4}\right)=0$;

(ii) $N\left(b, w_{1}\right)=N\left(b, w_{2}\right)=0, N\left(b, w_{3}\right)=N\left(b, w_{4}\right)=-1$;

(iii) $a$ and $b$ intersect only at $w$.

Now let $x=a b$, the curve obtained by transversing $a$ first and then $b$. As winding numbers are invariant under free homotopies of curves in $R$, we can deduce immediately that $x$ is not freely homotopic to any of the following curves:

(iv) a point in $R$;

(v) a simple closed curve;

(vi) a curve lying in any disc which contains exactly one of the punctures.

Now choose a point $z$ in $H$ over $w$ and lift the curves $a$ and $x$ from $z$ : this gives two curves $\tilde{a}$ and $\tilde{x}$ with terminal points $\alpha(x)$ and $\gamma(z)$ respectively (and $\tilde{a}$ is an initial segment of $\tilde{x}$ ). By (iv), $x$ (and hence $\tilde{x}$ ) is not homotopic to a point: thus $\gamma$ is not the identity. By (vi), $\gamma$ is not parabolic (else $\tilde{x}$ could be moved towards the fixed point of $\gamma$ and (vi) would be violated). Thus $\gamma$ is hyperbolic. Next, $\gamma$ is primitive, for suppose that $\gamma=\eta^{m}$ for some $\eta$ in $\Gamma$. Then $\eta$ is hyperbolic and $x$ is freely homotopic to a curve in $R$ transversed $m$ times, so $m$ divides each winding number $N\left(x, w_{j}\right)$. Thus $m= \pm 1$ and $\gamma$ is primitive. Finally, $\gamma$ is nonsimple, for otherwise $\tilde{x}$ could be deformed to a fundamental segment of $A_{\gamma}$ and then $x$ would violate $(\mathrm{v})$. Thus we have shown that $\gamma$ is a primitive nonsimple hyperbolic element of $\Gamma$.

Finally, we show that if $\beta\left(A_{\gamma}\right) \cap A_{\gamma} \neq \varnothing$, then $\beta$ is hyperbolic or the identity I. Construct the curve $L=\bigcup_{n \in \mathbf{Z}} \gamma^{n}(\tilde{x})$ with the fixed points of $\gamma$ adjoined (the final point of $\gamma^{n}(x)$ is the initial point of $\gamma^{n+1}(x)$, and because $\tilde{x}$ is compact, $L$ converges to the fixed points of $\gamma$ ). If we assume that $\beta\left(A_{\gamma}\right)$ crosses $A_{\gamma}$, then $\beta(L)$ crosses $L$. Thus there are points $u$ and $v$ on $\tilde{x}$ with $\beta \gamma^{r}(u)=\gamma^{s}(v)$. Because of (iii), the only $\Gamma$-equivalent points on $\tilde{x}$ are $z, \alpha(z)$ and $\gamma(z)$ : thus $\gamma^{-s} \beta \gamma^{r} \in$ $\left\{I, \alpha, \alpha^{-1}, \gamma, \gamma^{-1}, \gamma \alpha^{-1}, \alpha^{-1} \gamma\right\}$. It follows that there is a conjugate $\beta_{1}$ of $\beta$, or of $\beta^{-1}$, either in $\langle\gamma\rangle$ or in one of the cosets $\langle\gamma\rangle \alpha,\langle\gamma\rangle \alpha^{-1}$. If $\beta$ is parabolic, then so is 


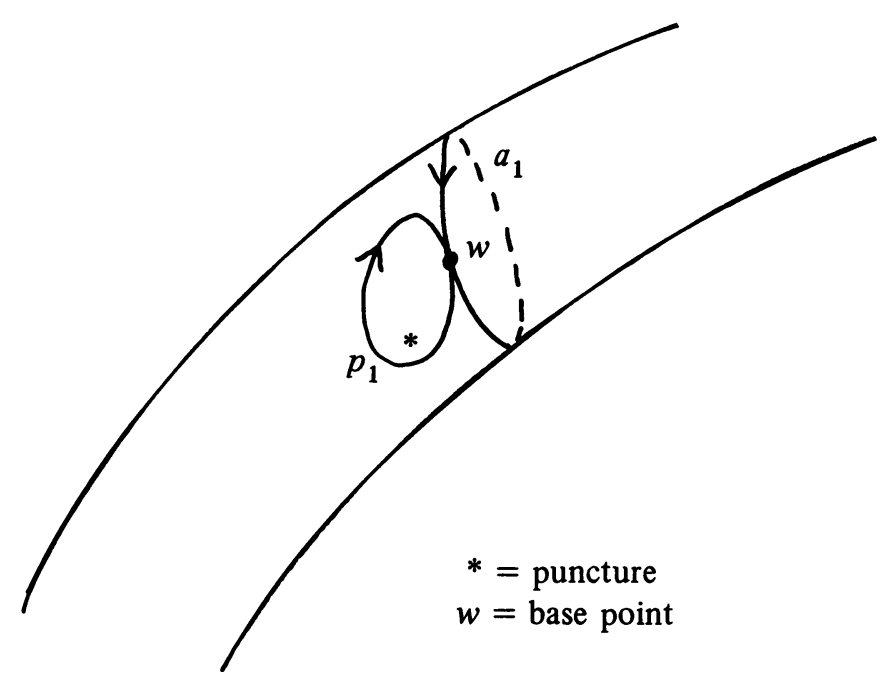

FIGURE 4

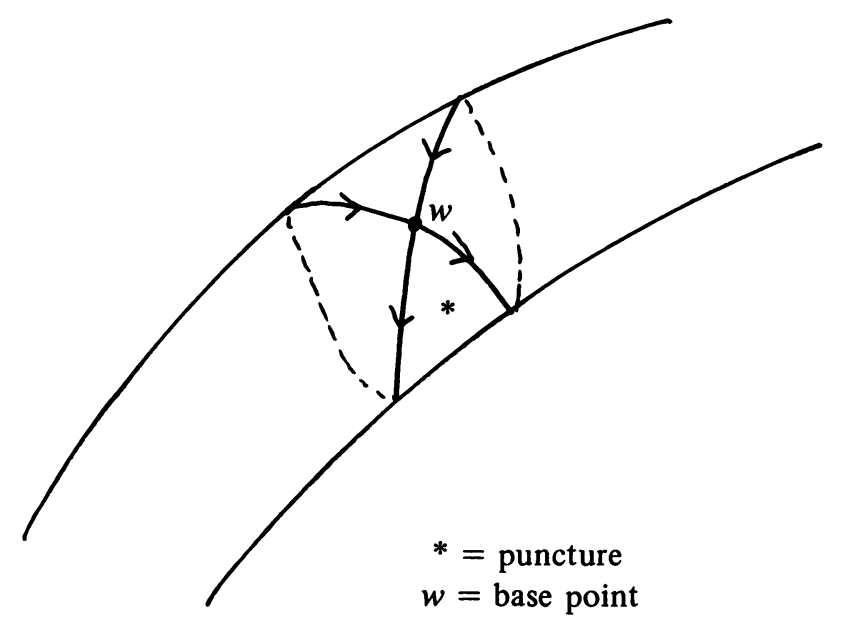

FIGURE 5

$\beta_{1}$, and since $\gamma$ is hyperbolic we find that $\beta_{1}=\gamma^{m} \alpha^{e}$ for some integer $m$ and some choice of $e= \pm 1$. We deduce that if $y=x^{m} a^{e}$, then $y$ is homotopic to some curve lying in a small neighborhood of one of the punctures. This is impossible, however, for

$$
N\left(y, w_{j}\right)=\left\{\begin{array}{cc}
m+e & \text { if } j=1,2, \\
-m & \text { if } j=3,4
\end{array}\right.
$$

and at least two of these four winding numbers must be nonzero. The proof for the case $g=0$ is complete.

The proof for $g \geq 1$. Clearly we may assume that $k \geq 1$ (else $\Gamma$ has no parabolic elements): thus we assume that $g \geq 1, k \geq 1$. In this part of the proof we use the standard presentation of the fundamental group $\Gamma^{*}$ of $R$ as the substitute for the 
winding number, namely

$$
\Gamma^{*}=\left\langle a_{1}, b_{1}, \ldots, a_{g}, b_{g}, p_{1}, \ldots, p_{k}: \prod_{i=1}^{g}\left[a_{i}, b_{i}\right] p_{k} \cdots p_{1}=I\right\rangle
$$

where $[a, b]=a b a^{-1} b^{-1}$ and the $p_{i}$ are parabolic. As $k \geq 1$, we can eliminate $p_{1}$ from the single relation in (4.1) and thereby realize $\Gamma^{*}$ as a free group on the free generators

$$
a_{1}, b_{1}, \ldots, a_{g}, b_{g}, p_{k}, \ldots, p_{2} .
$$

Every element of $\Gamma^{*}$ has a representation (not unique) as a word $W$ in the generators in (4.1) and a unique representation as a reduced word $W^{\prime}$ in the generators (4.2). $W^{\prime}$ is obtained from $W$ by replacing $\left(p_{1}\right)^{-1}$ by

$$
\prod_{i=1}^{g}\left[a_{i}, b_{i}\right] p_{k} \cdots p_{2}
$$

wherever it occurs in $W$ and then cancelling where possible.

Now let $\theta(u)$ be the sum of the exponents of $a_{1}$ in any word representing $u$. This sum is independent of the chosen word, and $\theta: \Gamma^{*} \rightarrow \mathbf{Z}$ is a homomorphism. Clearly, $\theta$ has the same value on conjugate elements of $\Gamma$, and, by definition, $\theta\left(p_{i}\right)=0$ for $j=1, \ldots, k$. It is well known that every parabolic element of $\Gamma$ is conjugate to some $p_{i}$, so $\theta(p)=0$ for every parabolic $p$ in $\Gamma$.

Now define

$$
x=\left(p_{1}\right)^{-1} a_{1} a_{1}=\prod_{i=1}^{g}\left[a_{i}, b_{i}\right] p_{k} \cdots p_{2} a_{1} a_{1},
$$

a reduced word with no $p_{i}$ present if $k=1$. We illustrate $a_{1}$ and $p_{1}$ in Figure 4 and a curve $y$ freely homotopic to $x$ in Figure 5 , all lying on a section of the $\left(a_{1}, b_{1}\right)$ handle of $R$ and beginning and ending at $w$.

As before, select $z$ over $w$ and lift $x$ to a curve $\tilde{x}$ from $z$ to $\gamma(z)$. It is now a matter of showing that $\gamma$ has the stated properties. Since $\theta(x)=2, \gamma$ is necessarily hyperbolic.

Next, we show that $\gamma$ is primitive. If not, then we can write

$$
x=\left(t_{1} \cdots t_{m}\right)^{s}=\left(t_{1} \cdots t_{m}\right) \cdots\left(t_{1} \cdots t_{m}\right)
$$

for some reduced word $t_{1} \cdots t_{m}$ and some $s \geq 2$. Because $\theta(x)=2$, we can only have $s=2$ (or $s=-2$, which is essentially the same case), and then

$$
\left[a_{1}, b_{1}\right] \cdots\left[a_{g}, b_{g}\right] p_{k} \cdots p_{2} a_{1} a_{1}=t_{1} \cdots t_{m} t_{1} \cdots t_{m} .
$$

The only cancellation possible on the right is $t_{m} t_{1}=I$ (the identity), then (possibly) $t_{m-1} t_{2}$, and so on. In any event, the word on the right when reduced begins with $t_{1}$ and ends with $t_{m}$. Since the word on the left of (4.5) is reduced, $t_{1}=a_{1}=t_{m}$, and so no cancellation was originally possible. In view of this, both words in (4.5) are already reduced, and we have a contradiction because $a_{1}$ occurs at least four times with exponent one on the right of (4.5).

Now let $\beta$ be a parabolic element of $\Gamma$. To show that $\beta\left(A_{\gamma}\right) \cap A_{\gamma}=\varnothing$, we assume the contrary and derive a contradiction. We replace $x$ by $y=\left(p_{1}^{-1} a_{1}\right)\left(a_{1}\right)$ (Figure 
2), constructed in the same free homotopy class and consisting of two simple loops meeting only at $w$. Lift $y$ and $z$ to obtain a curve $\tilde{y}$ from $z$ to $\gamma(z)$ (as $x$ and $y$ are freely homotopic) and construct $L\left(=\bigcup \gamma^{n}(\tilde{y})\right)$ as in the proof for $g=0$, using $\tilde{y}$ rather than $\tilde{x}$. Observe that the only distinct $\Gamma$-equivalent points on $\tilde{y}$ are $z, \alpha(z)$ and $\gamma(z)$. Exactly as before, we now obtain a conjugate $\beta_{1}$ of $\beta$ with $\beta_{1}=\gamma^{m} \alpha^{e}$, where $m$ and $e$ are integers and $e= \pm 1$. In this case we have

$$
0=\theta\left(\beta_{1}\right)=m \theta(\gamma)+e \theta(\alpha)=2 m \pm 1,
$$

which is a contradiction as $2 m \pm 1$ is an odd integer.

It remains only to show that $\gamma$ is nonsimple. We shall suppose that $\gamma$ is simple and again derive a contradiction. First, as $\theta(\alpha)=1$ and $\theta(\gamma)=2$, we see that $\alpha \notin\langle\gamma\rangle$. Thus $A_{\gamma}$ and $\alpha\left(A_{\gamma}\right)$ are disjoint. This means that the endpoints of $L$ and $\alpha(L)$ do not separate each other on the boundary of $H$, so as $\alpha(L)$ and $L$ cross at $\alpha(z)$, they must also cross at some other point $z^{\prime}$. We deduce that for some integers $m$ and $n, \gamma^{n}\left(z^{\prime}\right) \in \tilde{y}, \gamma^{m} \alpha^{-1}\left(z^{\prime}\right) \in \tilde{y}$. Thus either

(i) $\gamma^{n}\left(z^{\prime}\right)=\gamma^{m} \alpha^{-1}\left(z^{\prime}\right)$ or

(ii) $\gamma^{n}\left(z^{\prime}\right), \gamma^{m} \alpha^{-1}\left(z^{\prime}\right) \in\{z, \alpha(z), \gamma(z)\}$.

Now (i) is excluded as $\alpha \notin\langle\gamma\rangle$. From (ii) we deduce that $z^{\prime}$ lies in each of the sets

$$
\left\{\gamma^{-n}(z), \gamma^{-n} \alpha(z), \gamma^{1-n}(z)\right\}, \quad\left\{\alpha \gamma^{m}(z), \alpha \gamma^{-m} \alpha(z), \alpha \gamma^{1-m}(z)\right\}
$$

Of the nine possible identities obtained from the different possible choices of $z^{\prime}$, five are excluded because $\alpha \notin\langle\gamma\rangle$. The remaining possibilities yield

(iii) $\alpha^{-1} \gamma^{n} \alpha \gamma^{-m}=I$,

(iv) $\gamma^{n} \alpha \gamma^{-m} \alpha=I$,

(v) $\gamma^{n-1} \alpha \gamma^{-m} \alpha=I$

(vi) $\alpha^{-1} \gamma^{n} \alpha \gamma^{1-m}=I$.

Now any word in $\alpha$ and $\gamma$ when written in terms of the free generators (4.2) is automatically reduced for $\alpha$ corresponds to $a_{1}$ and $\gamma$ corresponds to $x$, which is itself reduced and which starts and ends with $a_{1}$. Thus (iv) and (v) cannot occir, and

(iii) implies that $m=n=0$,

(iv) implies that $n=0$ and $m=1$.

Returning to (4.6), we find that in each case $z^{\prime}=\alpha(z)$, so $\alpha(L)$ contains a loop from $\alpha(z)$ to $\alpha(z)$. Thus $L$ contains a loop from $z$ to $z$, and this cannot be because the only points on $L$ which are equivalent to $z$ are of the form $\gamma^{r} \alpha^{e}(z)$ with $e=0$ or 1 , and this is $z$ only when $e=r=0$. The proof is now complete.

5. Concluding remarks. The following modification of Theorem 1.1 is true (with essentially the same proof). Let $\Gamma$ be any finitely generated Fuchsian group: then $R(=H / \Gamma)$ is a surface $S$ of genus $g$ with $k$ punctures and $d$ discs removed. Corresponding to each of these $k+d$ components of the complement of $R$ in $S$, there is a conjugacy class of cyclic subgroups of $\Gamma$ (corresponding to the subgroup of the fundamental group of $R$ generated by a simple loop around the component). We call the elements in these subgroups the boundary elements of $\Gamma$ (every parabolic element of $\Gamma$ is of this type). Then, as suggested by Theorem 1.1 , we can select $\beta$ in (1.2) to be a boundary element of $\Gamma$ for every nonsimple $\gamma$ if and only if $g=0$ and $k+d=3$ or 4 . 


\section{BIBLIOGRAPHY}

1. H. Cohn, Approach to Markoff's minimal forms through modular functions, Ann. of Math. (2) 61 (1955), 1-12.

2. J. F. Koksma, Diophantische Approximationen, Chelsea, New York, n.d.

3. A. Haas, Diophantine approximation on hyperbolic Riemann surfaces, Acta Math. (Uppsala) (to appear).

4. J. Lehner and M. Sheingorn, Simple closed geodesics on $H^{+} / \Gamma(3)$ arise from the Markov spectrum, Bull. Amer. Math. Soc. (N.S.) 11 (1984), 359-362.

5. O. Perron, Die Lehre von den Kettenbrucken, Teubner, Stuttgart, 1954.

6. A. Schmidt, Minimum of quadratic forms with respect to Fuchsian groups. I, J. Reine Angew. Math. 286/7 (1976), 341-368.

7. C. Series, The geometry of Markoff numbers, Math. Intelligencer 7 (1985), 20-30.

8. M. Sheingorn, Characterization of simple closed geodesics on Fricke surfaces, Duke Math. J. 52 (1985), 535-545.

9. D. Zagier, On the number of Markov numbers below a given bound, Math. Comp. 39 (1982), 709-723.

Department of Mathematics, Cambridge University, Cambridge CB2 1SB, ENGLAND (Current address of A. F. Beardon)

School of Mathematics, institute for Advanced Study, Princeton, New JERSEY 08540

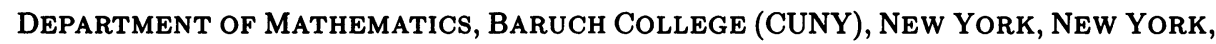
10010 (Current address of $M$. Sheingorn)

Current address (J. Lehner): 314-N Sharon Way, Jamesburg, New Jersey 08831 